\title{
B-factory Signals for a Warped Extra Dimension
}

\author{
Kaustubh Agashe ${ }^{* 1}$, Gilad Perez ${ }^{\dagger 2}$ and Amarjit Soni ${ }^{\# 3}$ \\ * Department of Physics and Astronomy, Johns Hopkins University, \\ Baltimore, MD 21218-2686 \\ $\dagger$ Theoretical Physics Group, Lawrence Berkeley National Laboratory, \\ Berkeley, CA 94720 \\ \# Brookhaven National Laboratory \\ Upton, NY 11973
}

\begin{abstract}
We study predictions for B-physics in a class of models, recently introduced, with a non-supersymmetric warped extra dimension. In these models few $(\sim 3) \mathrm{TeV}$ Kaluza-Klein masses are consistent with electroweak data due to bulk custodial symmetry. Furthermore, there is an analog of GIM mechanism which is violated by the heavy top quark (just as in SM) leading to striking signals at $B$-factories: (i) New Physics (NP) contributions to $\Delta F=2$ transitions are comparable to SM. This implies that, within this NP framework, the success of the SM unitarity triangle fit is a "coincidence". Thus, clean extractions of unitarity angles via e.g. $B \rightarrow \pi \pi, \rho \pi, \rho \rho, D K$ are likely to be affected, in addition to $O(1)$ deviation from SM prediction in $B_{s}$ mixing. (ii) $O(1)$ deviation from SM predictions for $B \rightarrow X_{s} l^{+} l^{-}$in rate as well as in forward-backward and direct CP asymmetry. (iii) Large mixing-induced CP asymmetry in radiative $B$ decays, wherein the SM unamibgously predicts very small asymmetries. Also, with KK masses $3 \mathrm{TeV}$ or less, and with anarchic Yukawa masses, contributions to electric dipole moments of the neutron are roughly 20 times larger than the current experimental bound so that this framework has a "CP problem".
\end{abstract}

Introduction. The standard model (SM) is in very good agreement with data. However, it is widely perceived to be an incomplete theory. In particular, in the SM, the hierarchy between the Planck scale and the electroweak symmetry breaking (EWSB) scale, $\Lambda_{\mathrm{EWB}}$, is unnatural since the Higgs mass is ultra-violet (UV) sensitive.

Solutions to the hierarchy problem, therefore, involve extending the SM at just above the EWSB scale which, in general, spoils the good agreement of the SM with data, for example, by introducing new sources of flavor violation. Given this inherent tension, it is important to identify new physics $[\mathrm{NP}]$ frameworks which preserve the appealing features of the SM.

We consider the Randall-Sundrum scenario (RS1) 1], which provides an elegant solution to the hierarchy problem, and show that this framework with mass scale of NP as low as a few TeV leads to many striking signals in Bphysics. This renders B-facilities a valuable probe of the parameter space of this novel physics scenario.

In this framework, due to warped higher-dimensional spacetime, the mass scales in an effective $4 D$ description depend on location in extra dimension: the Higgs sector is localized at the "TeV" brane where it is protected by a low warped-down fundamental scale of order a $\mathrm{TeV}$ while $4 D$ gravity is localized near the "Planck" brane which has

1 email: kagashe@pha.jhu.edu

2 email: gperez@lbl.gov

3 email: soni@quark.phy.bnl.gov a Planckian fundamental scale.

In the original RS1 model, the entire SM was localized on the $\mathrm{TeV}$ brane. In this set-up, flavor issues are sensitive to the UV completion of the RS1 effective field theory: there is no understanding of hierarchies in fermion masses or of smallness of flavor changing neutral currents (FCNC) from higher-dimensional operators which are naively too large being suppressed only by the warped-down cut-off $\sim \mathrm{TeV}$.

Allowing the SM fermions and gauge fields to propagate in the bulk makes flavor issues UV-insensitive as follows. The light fermions can be localized near the Planck brane (using a 5D fermion mass parameter [2, 3] ) where the effective cut-off is much higher than $\mathrm{TeV}$ so that FCNC's from higher-dimensional operators are suppressed [3, 4]. Moreover, this results in small 4D Yukawa couplings to the Higgs, even if there are no small $5 D$ Yukawa couplings [3, 4]. The top quark can be localized near $\mathrm{TeV}$ brane to obtain a large $4 D$ top Yukawa coupling. This provides an understanding of hierarchy of fermion masses (and mixing) without hierarchies in fundamental $(5 D)$ parameters solving the SM flavor puzzle.

With bulk fermions and gauge fields, FCNC's from exchange of gauge Kaluza-Klein (KK) modes are induced. The couplings of light fermions to gauge KK modes are flavor dependent (due to different wave-functions of 
fermions in the 5D), but, remarkably, this flavor dependence is small [5], since gauge KK modes are localized near the $\mathrm{TeV}$ brane, whereas light fermions are localized near the Planck brane (unlike the flat extra dimension case). Thus, FCNC's for light fermions from exchange of gauge KK modes are suppressed [3, 4]. Recall that this is the same reason why these fermions are light, i.e., the Higgs is also localized near $\mathrm{TeV}$ brane just like the gauge KK modes. Thus, this RS1 model has built-in ana$\log$ of the SM GIM mechanism and approximate flavor symmetries for the light fermions [6].

In spite of all these appealing features the above framework has another source of tension. It was shown that in order to be consistent with electroweak precision measurements (EWPM) one needs to assume $m_{\mathrm{KK}} \gtrsim$ $10 \mathrm{TeV}$ [7]. This brings a little hierarchy problem related to the smallness of $\left(\Lambda_{\mathrm{EWB}} / m_{\mathrm{KK}}\right)^{2} \sim 10^{-4}$, which is radiatively unstable.

Recently, it was shown that, with enhanced bulk electroweak (EW) gauge symmetry, $S U(2)_{L} \times S U(2)_{R} \times$ $U(1)_{B-L}$, few $\mathrm{TeV}$ KK masses are consistent with EWPM [8]. This gauge structure, with custodial symmetry, in warped space has also been used to construct Higgsless models of EWSB in [9, 10].

Our goal is to systematically study the flavor structure of the above framework and to re-investigate the NP contribution to FCNC processes [4] in the presence of rather light KK excitations. In this Letter, we will summarize the main effects and the ramifications for B-factories [ $[$ ]. Even though we mainly concentrate on RS1 model with the Higgs strictly localized on $\mathrm{TeV}$ brane, our results will be valid for warped space models without the Higgs or with 5D profile for Higgs as well. Thus these results are model independent within the new class of models which assume RS1 with custodial gauge symmetry in the bulk!

Flavor violation. Most of the flavor-violating effects are related to the violation of RS-GIM mechanism by large top quark mass (just as in SM) as follows. $(t, b)_{L}$ cannot be localized near the Planck brane. This is since the large top mass then requires a very large $5 D$ Yukawa coupling such that the theory is strongly coupled at the scale of the first KK mode [11]. Thus, $(t, b)_{L}$ must be localized near the $\mathrm{TeV}$ brane. This has two consequences: (1) In the interaction basis, the coupling of $b_{L}$ to gauge KK modes (say the gluons), $g_{G}^{b} \mathrm{KK}$, is large compared with the ones of the lighter quarks. This is a source of flavor violation which combined with mixing yields FCNC processes. (2) The Higgs VEV mixes zero and KK modes of $Z$ leading to a non-universal shift, in the interaction basis, of the coupling of $b_{L}$ to the physical $Z[8,12]$ :

$$
\delta g_{Z}^{b} \sim g_{Z^{\mathrm{KK}}}^{b} \sqrt{\log \left(M_{P l} / \mathrm{TeV}\right)} \frac{m_{Z}^{2}}{m_{\mathrm{KK}}^{2}},
$$

where $g_{Z^{\mathrm{KK}}}^{b}$ is the (non-universal) coupling between $b_{L}$ and a KK $Z$ state before EWSB. The factor of $\sqrt{\log \left(M_{P l} / \mathrm{TeV}\right)}$ comes from enhanced Higgs coupling to gauge KK mode (since they are localized near the $\mathrm{TeV}$ brane). EWPM, which are not related to flavor mixing, require that this shift is smaller than $\sim 1 \%$. Note that in the above framework the interaction basis is unique. This is the basis in which all the interactions (except Yukawa ones), including ones with KK modes are flavor diagonal.

There is, therefore, a tension between obtaining a large top Yukawa and not introducing a too large flavor violation in processes related to EWPM [8, 12]. As a result, all the models assume the following: (1) A large (close to maximal) dimensionless $5 D$ Yukawa, $\lambda_{5 D} \sim 4$ (such that the weakly coupled effective theory contains $3-4 \mathrm{KK}$ modes). (2) The wave function of $\left(t_{L}, b_{L}\right)$ is localized as close to the TeV brane as is allowed by EWPM so that a large (close to maximal) shift in coupling of $b_{L}$ to $Z$, $\delta g_{Z}^{b} \sim 1 \%$ is obtained. Using Eq. (1) we can summarize the above by the following $[\underline{6}]$

$$
g_{G^{\mathrm{KK}}}^{b} \sim g_{s}, \quad g_{Z^{\mathrm{KK}}}^{b} \sim g_{Z}, \quad m_{K K} \sim 3 \mathrm{TeV} .
$$

This (unavoidable) setup leads to sizable NP contributions in the following three kinds of top quark dominated FCNC processes: (i) $\Delta F=2$; (ii) $\Delta F=1$ governed by box and EW penguin diagrams; (iii) radiative decays. We will consider these effects, in turn, in what follows.

Let us elaborate more on how the NP contributions arise in the above framework. Since the couplings of fermions of a given type to gauge KK modes are nonuniversal, flavor mixing is induced when a transformation from the (unique) interaction to the mass basis is performed for the quarks. Here, we mostly consider couplings of left-handed down quarks. This is since coupling of $b_{L}$ to gauge KK modes is abnormally large [13] compared with the couplings of other down type quarks (which are localized near the Planck brane): the coupling of $b_{L}$ to gauge KK modes is "dictated" by $m_{t}$. Consequently a large RS-GIM violation is induced.

Signals. To study flavor-changing effects, we need to estimate the mixing angles of the unitary transformations. Generically 5D Yukawa matrices are expected to be anarchic. Thus the mixing angles of the transformations are roughly given by ratio of wave-functions at $\mathrm{TeV}$ brane. The unitary transformations for left-handed up, $U_{L}$, and down quarks, $D_{L}$, are similar due to the fact that $u_{L}$ and $d_{L}$ have the same wave-function. Since $U_{L} D_{L}^{\dagger}=V_{\mathrm{CKM}}$, we get $D_{L} \sim V_{\mathrm{CKM}}$. It follows, therefore, that the gluon KK mode- $b_{L^{-}}(s, d)_{L}$ vertex is roughly given by $g_{G^{\mathrm{KK}}}^{b} V_{t(s, d)}$ while the gluon KK mode- $d_{L^{-}} s_{L}$ one is $g_{G \mathrm{KK}}^{b} V_{t s} V_{t d}$.

This results in tree-level exchange of gluon KK mode contributing to $\Delta F=2$ operators:

$$
\frac{M_{12}^{\mathrm{RS}}}{M_{12}^{\mathrm{SM}}} \sim 16 \pi^{2} \frac{\left(g_{G^{\mathrm{KK}}}^{b}\right)^{2}}{g_{2}^{4}} \frac{m_{W}^{2}}{m_{\mathrm{KK}}^{2}} \sim C\left(g_{G^{\mathrm{KK}}}^{b}\right)^{2}\left(\frac{3 \mathrm{TeV}}{m_{\mathrm{KK}}}\right)^{2},
$$

where $C$ is an order one complex coefficient, mixing angles are of the same size in both RS1 and SM contri- 
butions and $M_{12}^{\mathrm{SM} \text {, RS }}$ is the SM (box diagram) and RS1 (KK gluon exchange) $\Delta F=2$ transition amplitudes respectively. We see that the KK gluon contribution to $B_{d}^{0}-\bar{B}_{d}^{0}, B_{s}^{0}-\bar{B}_{s}^{0}$ mass difference, $\epsilon_{K}$ and the CP asymmetry in $B \rightarrow \psi K_{S}$ is comparable to SM ones.

The SM predictions depend on $V_{t d}$ which is currently not severely constrained by tree-level decays and unitarity [14] which are not affected by NP contributions. The data, therefore, can be fitted even with RS1 contributions comparable to SM 6].

This, however, leads to a "coincidence problem": why is SM fit (usually presented as a plot of the constraints in the $\rho-\eta$ plane, see e.g. 14]) so good? At present, this problem is not so severe since there are $\mathcal{O}(20 \%)$ uncertainties in SM predictions for $\epsilon_{K}$ and $\Delta m_{B_{d}}$ (due mainly to hadronic matrix elements) [15] and also the RS1 contributions have $O(1)$ uncertainties due to fluctuations in $\lambda_{5 D}$. Consequently, clean measurements of $\alpha$ and $\gamma$ via $B \rightarrow \pi \pi, \pi \rho, \rho \rho, D K$ are likely to be affected.

The case of $B_{s}-\bar{B}_{s}$ mixing is slightly different than $B_{d}-\bar{B}_{d}$ mixing and $\epsilon_{K}$ as the SM contribution is known (up to hadronic matrix elements) since $V_{t s}$ is constrained by unitarity and tree-level decays [14]. Hence, for a generic order one complex coefficient (it is complex due to physical phases in $D_{L}[\underline{6}]$ ), we expect an $\mathcal{O}(1)$ deviation from SM prediction in $\Delta m_{B_{s}}$ (see reference [13] for a larger effect). Similarly, an $\mathcal{O}(1)$ time-dependent $\mathrm{CP}$ asymmetry in $B_{s} \rightarrow J / \psi \phi$ is induced compared with the SM $\mathcal{O}\left(\lambda_{c}^{2}\right)$ prediction, where $\lambda_{c} \sim 0.22$. Also deviations from SM expectation for $\gamma$ ought to occur in $B_{s} \rightarrow D K$.

Next, we consider $\Delta F=1$ transitions. We start with the discussion of processes which in the SM are dominated by QCD penguin diagrams such as $b \rightarrow s \bar{s} s$. There is a contribution from KK gluon exchange as in the $\Delta F=2$ case. The coupling of KK gluon to $s$ is suppressed by $\sim \sqrt{\log \left(M_{P l} / \mathrm{TeV}\right)}$ since the strange quark is localized near Planck brane whereas the KK gluon is localized near $\mathrm{TeV}$ brane (this is the universal part of coupling of fermions to gauge KK modes). Thus, it is clear that KK gluon contribution $\sim 1 / 5 \mathrm{SM}$ QCD penguin. In addition, there is dilution of $\mathrm{NP}$ effect in $\mathrm{QCD}$ penguin after RG scaling from $\mathrm{TeV}$ to $m_{b}$. So, KK gluon NP contributions in $\Delta F=1$ transition cannot compete with SM QCD penguin ones (see however 13] for a $O(1)$ effect with $\sim 1 \mathrm{TeV}$ KK masses).

The contribution from exchange of $K K$ mode of $Z$ is smaller than that from KK gluon. However, as mentioned above, the KK mode of $Z$ mixes with zero-mode of $Z$ due to Higgs vev, in turn, generating a flavor-violating coupling to the physical $Z$. Thus, we get the following contributions to coefficients of EW penguin operators, $C_{7-10}$ (four quark) and $C_{9 V, 10 A}$ (leptonic operators):

$$
\frac{C_{7-10,9 V, 10 A}^{Z, \mathrm{RS}}}{C_{7-10,9 V, 10 A}^{Z, \mathrm{SM}}} \sim \frac{16 \pi^{2}}{g_{2}^{2}} \frac{g_{Z \mathrm{KK}}^{b}}{g_{Z}} \sqrt{\log \left(M_{P l} / \mathrm{TeV}\right)} \frac{m_{Z}^{2}}{m_{\mathrm{KK}}^{2}}
$$

$$
\sim \frac{g_{Z \mathrm{KK}}^{b}}{g_{Z}}\left(\frac{3 \mathrm{TeV}}{m_{\mathrm{KK}}}\right)^{2}
$$

where superscript $Z$ on $C_{7-10,9}, 10 A$ denotes $Z$ penguin part and, as for $\Delta F=2$ case, the SM contribution is from top quark loop and mixing angles are of same size in both contributions. Thus, the two contributions are comparable. This leads to an $\mathcal{O}(1)$ effect in BR for rare flavor-changing decays, for example, $b \rightarrow s l^{+} l^{-}$16 12, where uncertainty in theory prediction is of $\mathcal{O}(20 \%)$ and current experimental error (from BABAR and BELLE) is of $\mathcal{O}(30 \%)$ 17. In addition a smoking gun signal is that significant departure from SM is expected in the direct CP asymmetry and in the spectrum of leptons in this decay, as well as in the forward-backward asymmetry since the new physics effect is only in the $Z$ penguin (with almost axial couplings of leptons, i.e., in $C_{10 A}$ ) and not in the photon penguin (with vector couplings of leptons, i.e., in $C_{9 V}$ ).

As mentioned above, the $b \rightarrow s \bar{s} s$ transition is dominated by SM QCD penguin. Similar RS1 contribution from flavor-violating $Z$ vertex is suppressed by at least $\sim g_{Z}^{2} / g_{s}^{2} \sim 20 \%$ and therefore subleading [16] 12. Consequently, RS1 can accomodate only mild deviations $[\underline{6}$ (unlike 13], as mentioned above) from the SM in time dependent $\mathrm{CP}$ asymmetry in "penguin-doiminated" $\mathrm{B}$ decays, such as $\phi\left(\eta^{\prime}, \pi^{0}, \omega, \rho^{0}\right) K_{s}$.

We next consider radiative decay processes. Since these require helicity flip, related NP contributions appear only at the loop level in our framework. The dominant contribution comes from loops of Higgs and $K K$ fermions since couplings of KK fermions to Higgs are enhanced. We show elsewhere [6] that KK gluon contribution is aligned in flavor space with fermion mass matrix and hence is not flavor-violating. We find the following contribution to dipole operator for $b \rightarrow s$ transition:

$$
\begin{aligned}
\frac{C_{7 \gamma}^{\prime \mathrm{RS}}}{C_{7 \gamma}^{\mathrm{SM}}} & \sim \frac{\lambda_{5 D}^{2}}{g_{2}^{2}} \frac{m_{W}^{2}}{m_{K K}^{2}} \frac{\left(D_{R}\right)_{23}}{V_{t s}} \\
& \sim\left(D_{R}\right)_{23}\left(\frac{\lambda_{5 D}}{4}\right)^{2}\left(\frac{3 \mathrm{TeV}}{m_{K K}}\right)^{2},
\end{aligned}
$$

where $C_{7 \gamma}$ and $C_{7 \gamma}^{\prime}$ are coefficients of dipole operators with $b_{R}$ and $b_{L}$, respectively and $\left(D_{R}\right)_{23} \rightarrow\left(D_{L}\right)_{23}$ for $C_{7 \gamma}^{R S}$. For $b \rightarrow d$ transition, $\left(D_{L}, R\right)_{23} \rightarrow\left(D_{L}, R\right)_{13}$ and $V_{t s} \rightarrow V_{t d}$.

Let us now estimate the right-handed $(\mathrm{RH})$ down quark mixing appearing in the above RS1 contribution. Due to anarchic $\lambda_{5 D}$, the ratio of masses are also given by ratio of wave-functions on $\mathrm{TeV}$ brane (just like the mixing angles) so that:

$$
\frac{m_{s}}{m_{b}}\left(D_{L}\right)_{23}^{-1} \sim\left(D_{R}\right)_{23} \sim \frac{m_{s}}{m_{b}} V_{t s}^{-1}=\mathcal{O}(1),
$$

where we used the bottom and strange quark masses at the $\sim$ TeV scale and also $\left(D_{L}\right)_{23} \sim V_{t s}$. Similarly, we 
find $\left(D_{R}\right)_{13} \sim \lambda_{c}$, i.e., $\mathrm{RH}$ down quark mixing are much larger than left-handed. Then, from Eq. (5), we see that RS1 contribution to $b_{L} \rightarrow(s, d)_{R} \gamma$ is comparable to SM contribution to $b_{R} \rightarrow(s, d)_{L} \gamma$ for $\lambda_{5 D} \sim 4$. Also, NP contribution to $b_{R} \rightarrow(s, d)_{L} \gamma$ is negligible (see reference [18] for an earlier study of only this operator).

This leads to another smoking gun signal: $\mathcal{O}(1)$ mixing induced CPV due to interference between the SM amplitude for $b_{R} \rightarrow(s, d)_{L} \gamma$ and the NP contribution to $b_{L} \rightarrow(s, d)_{R} \gamma$ [19] and also deviation from a pure left handed polarization of the emitted photon [20]. This will be tested in $B \rightarrow K^{*} \gamma, B_{s} \rightarrow \phi \gamma(b \rightarrow s)$ transitions and $B \rightarrow \rho \gamma, B_{s} \rightarrow K^{*} \gamma(b \rightarrow d)$ transitions.

Finally, we discuss contribution to neutron's electric dipole moment (EDM) which arise from similar diagrams. With $O(1)$ complex phases, the contribution exceeds the experimental limits by $\mathcal{O}(20)$. We find that while contributions from CKM-like phases vanish at the one loop level sizable contributions are induced by Majorana-like phases. Though this requires flavor mixing, even with two flavors we find unsuppressed one loop amplitudes [6]. Thus, our framework has a CP problem!

Conclusions. Within the RS1 framework, localization of light fermions far from the $\mathrm{TeV}$ brane leads to three virtues: (i) Suppression of higher dimensional flavor violating operators. (ii) Suppression of flavor violating coupling to KK excitations. (iii) A solution to the SM flavor puzzle. There is a built-in analog of GIM mechanism of the SM and approximate flavor symmetries. As in SM, inclusion of heavy top quark leads to RS-GIM violation, in particular, to large couplings of left-handed bottom to gauge KK modes, in turn, resulting in $O(1)$ effects in $\Delta F=2$ processes and in rare flavor- changing decays, for example, $b \rightarrow s l^{+} l^{-}$. Also, the large $5 D$ Yukawa required to obtain top mass coupled with large $\mathrm{RH}$ down quark mixing leads to $O(1)$ effect in radiative $B$ decays. These B-physics signals should be of great relevance to $\mathrm{B}$-facilities in hadronic and in $e^{+} e^{-}$environments. Finally, the above framework suffers from a CP problem.

Using the AdS/CFT correspondence, the weaklycoupled RS1 model can be viewed as a tool to study a purely $4 D$ strongly coupled conformal Higgs sector 21. Thus, a key point of our study is that a $4 D$ strongly interacting Higgs sector can solve the flavor puzzle and have suppressed FCNC's with striking signals (see Table $1)$ at $B$ facilities.

Acknowledgements. K.A. is supported by the Leon Madansky fellowship \& NSF Grant P420D3620414350; G.P. \& A.S. are supported by DOE under Contract Nos. DE-AC0376SF00098 \& DE-AC02-98CH10886. We thank G. Burdman, Z. Chacko, W. Goldberger, Y. Grossman, I. Hinchliffe, D. E. Kaplan, Y. Nir, Y. Nomura, F. Petriello, R. Sundrum \& M. Suzuki for discussions.
[1] L. Randall and R. Sundrum, Phys. Rev. Lett. 83, 3370 (1999) arXiv:hep-ph/9905221.

[2] Y. Grossman and M. Neubert, Phys. Lett. B 474, 361 (2000) arXiv:hep-ph/9912408.

[3] T. Gherghetta and A. Pomarol, Nucl. Phys. B 586, 141 (2000) arXiv:hep-ph/0003129.

[4] For earlier work assuming $\sim 10 \mathrm{TeV}$ KK masses, see: S. J. Huber and Q. Shafi, Phys. Lett. B 498, 256 (2001) arXiv:hep-ph/0010195; G. Burdman, Phys. Rev. D 66, 076003 (2002) arXiv:hep-ph/0205329; S. J. Huber, Nucl. Phys. B 666, 269 (2003) arXiv:hep-ph/0303183; S. Khalil and R. Mohapatra, arXiv:hep-ph/0402225

[5] There is a sizable flavor-universal part of this coupling.

[6] For further details see, K. Agashe, G. Perez and A. Soni, in preparation.

[7] S. J. Huber and Q. Shafi, Phys. Rev. D 63, 045010 (2001) arXiv:hep-ph/0005286; S. J. Huber, C. A. Lee and Q. Shafi, Phys. Lett. B 531, 112 (2002) arXiv:hep-ph/0111465; C. Csaki, J. Erlich and J. Terning, Phys. Rev. D 66, 064021 (2002) arXiv:hep-ph/0203034; J. L. Hewett, F. J. Petriello and T. G. Rizzo, JHEP 0209, 030 (2002) arXiv:hep-ph/0203091.

[8] K. Agashe et al., JHEP 0308, 050 (2003) arXiv:hep-ph/0308036.

[9] C. Csaki et al., Phys. Rev. Lett. 92, 101802 (2004) arXiv:hep-ph/0308038 and arXiv:hep-ph/0310355

[10] Y. Nomura, JHEP 0311, $050 \quad$ (2003) arXiv:hep-ph/0309189.

[11] There is also a limit on how close $t_{R}$ can be to the TeV brane since its custodial isospin partner becomes too light which leads to phenomenological problems. Thus this cannot be used to enhance the top Yukawa coupling [8].

[12] G. Burdman and Y. Nomura, arXiv:hep-ph/0312247

[13] For an earlier RS1 study with low KK masses, see G. Burdman, Phys. Lett. B 590, 86 (2004) arXiv:hep-ph/0310144. It differs from our work since hierarchies in the $5 D$ Yukawas were allowed and constraints from $Z \rightarrow b \bar{b}$ were not correlated with the FCNC analysis.

[14] K. Hagiwara et al. [Particle Data Group Collaboration], Phys. Rev. D 66, 010001 (2002).

[15] G. Eyal, Y. Nir and G. Perez, JHEP 0008, 028 (2000) arXiv:hep-ph/0008009.

[16] K. Agashe, Workshop on a B Factory at $10^{36}$ Luminosity, SLAC, (Oct, 2003) [to appear in proceedings].

[17] T. Hurth, Rev. Mod. Phys. 75, 1159 (2003) arXiv:hep-ph/0212304; A. Ghinculov et al., Nucl. Phys. B 685, 351 (2004) arXiv:hep-ph/0312128; for a recent experimental review see: M. Nakao, arXiv:hep-ex/0312041

[18] C. S. Kim et al., Phys. Rev. D 67, 015001 (2003) arXiv:hep-ph/0204002.

[19] D. Atwood, M. Gronau and A. Soni, Phys. Rev. Lett. 79, 185 (1997) arXiv:hep-ph/9704272 ; B. Aubert et al. [BABAR Collaboration], arXiv:hep-ex/0405082

[20] M. Gronau et al, Phys. Rev. Lett. 88, 051802 (2002) arXiv:hep-ph/0107254.

[21] N. Arkani-Hamed, M. Porrati and L. Randall, JHEP 0108, 017 (2001) arXiv:hep-th/0012148. 


\begin{tabular}{|c|c|c|c|c|c|c|}
\hline & $\Delta m_{B_{s}}$ & $S_{B_{s} \rightarrow \psi \phi}$ & $S_{B_{d} \rightarrow \phi K_{s}}$ & $B r\left[b \rightarrow s l^{+} l^{-}\right]$ & $S_{B_{d, s} \rightarrow K^{*}, \phi \gamma}$ & $S_{B_{d, s} \rightarrow \rho, K^{*} \gamma}$ \\
\hline \hline $\mathrm{RS} 1$ & $\Delta m_{B_{s}}^{\mathrm{SM}}[1+O(1)]$ & $O(1)$ & $\sin 2 \beta \pm O(.2)$ & $B r^{\mathrm{SM}}[1+O(1)]$ & $O(1)$ & $O(1)$ \\
\hline $\mathrm{SM}$ & $\Delta m_{B_{s}}^{\mathrm{SM}}$ & $\lambda_{c}^{2}$ & $\sin 2 \beta$ & $B r^{\mathrm{SM}}$ & $\frac{m_{s}}{m_{b}}\left(\sin 2 \beta, \lambda_{c}^{2}\right)$ & $\frac{m_{d}}{m_{b}}\left(\lambda_{c}^{2}, \sin 2 \beta\right)$ \\
\hline
\end{tabular}

TABLE I: Contrasting signals from RS1 with the SM 